See Article page $\mathrm{XXX}$.

\section{Commentary: Heart failure and the problem of causality}

\author{
Keshava Rajagopal, MD, $\mathrm{PhD}$, \\ Faisal H. Cheema, MD, ${ }^{a, b, c}$ and Shuab Omer, MD ${ }^{a}$
}

\section{"The cause is hidden, but the result is known."- Ovid, Metamorphoses}

Causality, or the relationship linking cause to process, and thence to effect, is one of the pillars of scientific thought. In medicine, causality links etiology, pathogenesis, and clinical manifestation. In this issue of the Journal, Duggal and colleagues ${ }^{1}$ report the results of transcriptional ("transcriptomic") profiling of end-stage heart failure in the presence or absence of significant mitral valve regurgitation (MR). Briefly summarized, they compared left ventricle (LV) apical specimens from patients with either moderate or greater MR or moderate MR or less at the time of continuous-flow LV assist device implantation, versus LV apical specimens from potential donor hearts with normal LV systolic function that were unused for cardiac transplantation. They found that although substantial transcriptional profile overlap was present across all end-stage heart failure LVs, the presence or absence of many gene transcripts was differentially associated with either moderate or less MR or moderate or greater MR. The authors state, "MR is associated with specific transcriptomic signatures in patients undergoing LV assist device implant," and "significant MR increased immune transcripts and decreased structural gene expression." Yet, as we shall discuss, such conclusions are stated on shaky logical grounds.

The first issue to be addressed concerns physiological causality. It is well-known and understood that LV dysfunction and MR are interrelated in terms of cause and effect.

\footnotetext{
From the ${ }^{\mathrm{a}}$ Houston Heart, HCA Houston Healthcare, Houston, Tex; ${ }^{\mathrm{b}}$ Department of Clinical Sciences, University of Houston College of Medicine, Houston, Tex; and ${ }^{\mathrm{c}} \mathrm{HCA}$ Research Institute, Nashville, Tenn.

Disclosures: The authors reported no conflicts of interest.

The Journal policy requires editors and reviewers to disclose conflicts of interest and to decline handling or reviewing manuscripts for which they may have a conflict of interest. The editors and reviewers of this article have no conflicts of interest.

Received for publication Sept 2, 2021; revisions received Sept 2, 2021; accepted for publication Sept 2, 2021.

Address for reprints: Shuab Omer, MD, Houston Heart, HCA Houston Healthcare, 1200 Binz St, Suite 900, Houston, TX 77004 (E-mail: shueyb@yahoo.com). J Thorac Cardiovasc Surg 2021; $\mathbf{\square}: 1-2$ $0022-5223 / \$ 36.00$

Copyright (C) 2021 by The American Association for Thoracic Surgery https://doi.org/10.1016/j.jtcvs.2021.09.002
}

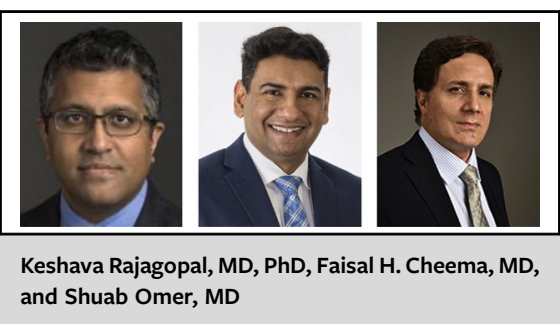

CENTRAL MESSAGE

The existence and mechanisms of causality are of primary importance in science.

MR of any morphology may cause LV dilatation and eventual systolic dysfunction; conversely, LV systolic dysfunction in the initial absence of MR may cause mitral valve and apparatus remodeling and subsequent "functional" MR (which may exacerbate LV dysfunction). The authors' observations and conclusions in this context seem to fit. And yet, regardless of molecular mechanism (see to follow), the heart is an organ that serves a mechanical function. Why are no differences whatsoever observed (or at least reported) with respect to cardiovascular parameters between the moderate or less MR and moderate or greater MR groups, with the sole exception of tricuspid valve regurgitation? That is to say, what is the meaning of such MR classifications, if there are no demonstrable physiological effects? Further, what then would be the physiological meaning and causal importance of the transcriptomic findings observed? One might counter that the absence of hemodynamic differences between the 2 groups means that the transcriptomic findings are more specifically related to MR. However, this still concedes that the MR is not particularly important physiologically, and there yet remains the possibility that "moderate or greater" but physiologically unimportant $\mathrm{MR}$ is present, and the transcriptomic findings are unrelated to MR but related to other variables associated-causally or not-with MR. For example, the findings might actually relate to tricuspid valve regurgitation and secondary hepatic dysfunction. In addition, the weights of the patients with moderate or greater MR were substantially lower; are the transcriptomic findings a readout of cardiac cachexia?

The second issue to be addressed concerns molecular causality. Pathologic as well as adaptive feedback stimuli in heart failure, typically via engagement of cell-surface receptors, initiate, stimulate, suppress, or arrest intracellular signal transduction pathways. The most common end-result of modulation of cell surface receptor-mediated signaling 
and downstream effector pathways is modulation of gene transcription. Readouts of gene transcription alterations accordingly may be results of pathological or feedback adaptive stimuli, or may be the result of pathologic or feedback adaptive intracellular signal transduction. That is to say, the findings observed could be related to a pathologic process or an adaptation to a pathological process. Moreover, even if the upstream signals themselves are pathological, the transcriptional responses might be adaptive (or vice versa). Finally, although not necessarily related to molecular causality, but yet related to mechanism, transcript levels may or may not translate (literally) to peptide/protein levels, which may or may not correlate with the functionality or activity of these peptides/proteins.

The study of Duggal and colleagues ${ }^{1}$ is thorough and thought-provoking. However, the findings need to be interpreted cautiously. Causality and mechanism are vitally important concepts that we must continue to use in scientific research.

\section{Reference}

1. Duggal NM, Lei I, Wu X, Aaronson KD, Pagani FD, Lam HYK, et al. Mitral regurgitation severity at LVAD implantation is associated with distinct myocardial transcriptomic signatures. J Thorac Cardiovasc Surg. September 14, 2021 [Epub ahead of print]. 